\title{
Entanglement estimation from Bell inequality violation
}

\author{
Karol Bartkiewicz, ${ }^{1,2, \text { f }}$ Bohdan Horst, ${ }^{2}$ Karel Lemr, ${ }^{1}$ and Adam Miranowicz ${ }^{2}$ \\ ${ }^{1}$ RCPTM, Joint Laboratory of Optics of Palacky University and \\ Institute of Physics of Academy of Sciences of the Czech Republic, \\ 17. listopadu 12, 772 07 Olomouc, Czech Republic \\ ${ }^{2}$ Faculty of Physics, Adam Mickiewicz University, 61-614 Poznań, Poland
}

\begin{abstract}
It is well known that the violation of Bell's inequality in the form given by Clauser, Horne, Shimony, and Holt (CHSH) in two-qubit systems requires entanglement, but not vice versa, i.e., there are entangled states which do not violate the CHSH inequality. Here we compare some standard entanglement measures with violations of the CHSH inequality (as given by the Horodecki measure) for two-qubit states generated by Monte Carlo simulations. We describe states that have extremal entanglement according to the negativity, concurrence, and relative entropy of entanglement for a given value of the CHSH violation. We explicitly find these extremal states by applying the generalized method of Lagrange multipliers based on the Karush-Kuhn-Tucker conditions. The found minimal and maximal states define the range of entanglement accessible for any two-qubit states that violate the CHSH inequality by the same amount. We also find extremal states for the concurrence versus negativity by considering only such states which do not violate the CHSH inequality. Furthermore, we describe an experimentally efficient linear-optical method to determine the highest Horodecki degree of the CHSH violation for arbitrary mixed states of two polarization qubits. By assuming to have access simultaneously to two copies of the states, our method requires only six discrete measurement settings instead of nine settings, which are usually considered.
\end{abstract}

PACS numbers: 03.67.Mn, 03.65.Ud, 42.50.Dv

\section{INTRODUCTION}

Since the seminal paper of Einstein, Podolsky and Rosen [1, there has been much interest in the two seemingly interrelated phenomena of quantum entanglement and nonlocality. Especially during the last three decades much theoretical and experimental work has been done in order to better understand the implications of these phenomena not only in physics but even in biology and philosophy.

Quantum entanglement is nowadays relatively well understood [2]. It is defined as the inseparability of quantum states and can be viewed as an algebraic concept. Quantum nonlocality is more related to experimental statistics. Namely, it can be considered as a type of correlation between measurement outcomes, obtained in spatially and temporally separated laboratories, that cannot be explained by local hidden-variable theories. Bell-type inequalities [3, 4] are often used to address this nonlocality quantitatively 5]. In this paper we focus on the violation of Bell's inequality in the form derived by Clauser, Horne, Shimony, and Holt (referred to as the CHSH inequality) 4.

For two qubits, Bell inequalities can be violated only if their states are entangled. However, as shown by Werner [6], there are entangled states that can still exhibit correlations which do not violate any Bell inequality for any possible local measurements; that is, unless a sequence of measurements, or several copies, or other more

*Electronic address: bartkiewicz@jointlab.upol.cz sophisticated scenarios are applied [5]. Note that Werner considered only projective measurements, but his conclusions apply also to the case of general measurements [positive operator-valued measures POVMs] [5].

Werner's states are defined as 6]

$$
\hat{\rho}_{W}(p)=p\left|\Psi^{-}\right\rangle\left\langle\Psi^{-}\right|+\frac{1-p}{4} I \otimes I
$$

which is a mixture of the singlet state $\left|\Psi^{-}\right\rangle=(|01\rangle-$ $|10\rangle) / \sqrt{2}$ and the maximally mixed state $I \otimes I$, where $I$ is the single-qubit identity operator and the parameter $p \in[0,1]$. The Werner states violate the $\mathrm{CHSH}$ inequality if and only if $1 / \sqrt{2}<p \leq 1$, while they are entangled iff $1 / 3<p \leq 1$. Thus, for $p \in(1 / 3,1 / \sqrt{2}]$, the Werner states are entangled and they satisfy the CHSH inequality 6]. These properties of the Werner states can be easily revealed by applying the Horodecki theorem [7.

Therefore, a natural question can be raised as to how much entangled states can be without violating the CHSH inequality or, more generally, for any fixed degree of the $\mathrm{CHSH}$ violation. The intuitive guess is that different measures of entanglement imply different answers for this question.

A degree of entanglement of two-qubit states can be described by various entanglement measures including [2] (i) the relative entropy of entanglement (REE) [8], which is a quantum version of the Kullback-Leibler divergence; (ii) the Peres-Horodecki negativity [9], which is a measure of the entanglement cost under operations preserving the positivity of partial transpose (PPT) 10; and (iii) the Wootters concurrence [11, a measure of the entanglement of formation [12. On the other hand, the Horodecki theorem [7] enables not only testing the $\mathrm{CHSH}$ inequality violation but also quantifying the degree of this violation 
for arbitrary two-qubit states. This degree is often referred to as a single-copy nonlocality measure [5].

In this paper we shall use the listed measures of entanglement to answer the question about the relation between the CHSH violation and entanglement quantitatively. In particular we find states that have extreme entanglement for all the above-mentioned entanglement measures for a given degree of the CHSH violation. For the purpose of our optimization procedure we shall use the so-called Karush-Kuhn-Tucker (KKT) conditions in a generalized method of Lagrange multipliers, which provide powerful tools for solving such optimization problems [13. We also use other tools for testing the optimality of the states obtained, namely, the optimality conditions for the concurrence provided in Ref. 14] and Monte Carlo simulations.

Verstraete and Wolf 14 found the regions of possible extremal CHSH violation for a given concurrence. This comparison is an important result, but it does not indicate the regions of extremal $\mathrm{CHSH}$ violation for other important entanglement measures including the negativity and REE. For example, in contrast to the VerstraeteWolf results, pure states are not extremal if the CHSH violation is compared with the REE (for values not too close to 1) as recently shown in Ref. [15. Here we give a deeper comparison of the REE and CHSH violation. More importantly, we find the regions of the extremal negativity for a given CHSH violation.

The inequivalence of such results for different entanglement measures in comparison to the $\mathrm{CHSH}$ violation can be understood by recalling that these measures have fundamentally different physical meanings (as discussed in Sec.20 even for two-qubit states. Only in special cases, including pure states, the negativity and concurrence become equal and equivalent to the REE. As an example of such basic discrepancies, we will show explicitly that these three entanglement measures do not necessarily imply the same ordering of states even if the CHSH violation is fixed at some value. Actually, as shown in Ref. [16], all "good" nonidentical asymptotic entanglement measures (such as those studied in this paper) cannot impose consistent orderings for all quantum states.

We study the relation between the CHSH violation and negativity (and other entanglement measures) for arbitrary two-qubit states analogously to the comparisons of the CHSH violation with the concurrence [14, 17] and REE [15. Note that many other comparative studies of the concurrence and CHSH violation were limited to some specific classes of two-qubit states usually in a dynamical context 18 26.

It is worth noting an increasing interest in developing device-independent approaches to entanglement testing and quantifying, which are based on various Bell inequality violations (see, e.g., Ref. [5, 27] and references therein). For example, semi-device-independent upper and lower bounds on the concurrence were studied in Ref. 28, and device-independent lower bounds on the negativity were found recently in Ref. 27]. These ap- proaches often correspond to testing only sufficient conditions for the CHSH violation. They are, however, beyond the scope of this work, which is focused on the maximal violations of the CHSH inequality based on the necessary and sufficient conditions as described by the Horodecki measure. For example, Ref. [27] employed some methods and results obtained in the studies of matrices of moments for continuous variable systems related to the criteria (i.e., witnesses instead of measures) of entanglement 29] and nonclassicality [30].

We focus on the CHSH inequality, although there are stronger Bell inequalities as was shown for the Werner states by, e.g., Vertesi 31. Note, however, that the CHSH inequalities even though simple (there is a variety of other Bell inequalities involving more measurement settings) are very powerful since the stronger inequality requires at least 465 settings on each side for the Vertesi inequality. The Werner states violate the Vertesi inequality for $p>0.7056$, while the CHSH inequality is violated, as already mentioned for $p>1 / \sqrt{2} \sim 0.7071$ only. There are other Bell inequalities that are not equivalent to the ones already mentioned. Notably, there is the Śliwa-Collins-Gisin inequality [32, i.e., the so-called $I_{3322}$ inequality.

Furthermore, we describe an efficient experimental method for estimating the Horodecki measure of the CHSH violation for two polarization qubits in an unknown arbitrary state. By assuming we have access simultaneously to two copies of the state, we show how to perform such a measurement with only six discrete measurement settings for estimating the Horodecki measure. Of course, with an a priori (even partial) knowledge of a given state or, in particular, of the optimal experimental settings for its detection, one can further simplify the proposed method. For example, optimized experimental settings for the best measurement of the CHSH violation for an a priori known class of two-qubit states were studied in Ref. 33].

The paper is organized as follows. In Sec. 2 we review some basic definitions used throughout the paper. In the following Sec. 3 we provide the boundary states for a given value of the CHSH violation and analytic expressions for their entanglement in terms of the degree of CHSH violation. The extremality conditions for the negativity and concurrence versus the $\mathrm{CHSH}$ violation are tested in Sec. 4 and Appendix A, respectively. In Sec. 5 we compare the concurrence and negativity for states satisfying the CHSH inequality. Section 6 presents a description of an experimental proposal for measuring the maximal $\mathrm{CHSH}$ violation degree using the same six settings regardless of the investigated two-qubit state. We conclude in Sec. 7 .

\section{PRELIMINARIES}

Throughout this paper we study correlations in twoqubit systems described by density matrices $\rho$, which can 
be expressed in the standard Bloch representation as follows

$$
\rho=\frac{1}{4}\left(I \otimes I+\vec{x} \cdot \vec{\sigma} \otimes I+I \otimes \vec{y} \cdot \vec{\sigma}+\sum_{n, m=1}^{3} T_{n m} \sigma_{n} \otimes \sigma_{m}\right),
$$

where the correlation matrix $T_{i j}=\operatorname{Tr}\left[\rho\left(\sigma_{i} \otimes \sigma_{j}\right)\right]$, and the vectors $\vec{\sigma}=\left[\sigma_{1}, \sigma_{2}, \sigma_{3}\right]$, and $\vec{x}(\vec{y})$ with elements $x_{i}=$ $\operatorname{Tr}\left[\rho\left(\sigma_{i} \otimes I\right)\right]\left(y_{i}=\operatorname{Tr}\left[\rho\left(I \otimes \sigma_{i}\right)\right]\right)$, are expressed in terms of the Pauli matrices. As discussed further in the text, this form of two-qubit density matrix is very convenient for investigating the $\mathrm{CHSH}$ violation.

\section{A. Measure of $\mathrm{CHSH}$ violation}

The CHSH inequality for a two-qubit state $\rho \equiv \rho_{A B}$ can be written as [2, 4]:

$$
\left|\operatorname{Tr}\left(\rho \mathcal{B}_{\mathrm{CHSH}}\right)\right| \leq 2
$$

in terms of the CHSH operator

$$
\mathcal{B}_{\mathrm{CHSH}}=\vec{a} \cdot \vec{\sigma} \otimes\left(\vec{b}+\vec{b}^{\prime}\right) \cdot \vec{\sigma}+\vec{a}^{\prime} \cdot \vec{\sigma} \otimes\left(\vec{b}-\vec{b}^{\prime}\right) \cdot \vec{\sigma}
$$

where $\vec{a}, \vec{a}^{\prime}$ and $\vec{b}, \vec{b}^{\prime}$ are unit vectors describing the measurements (i.e., detector settings) on sides $A$ (Alice) and $B$ (Bob), respectively. As shown by Horodecki et al. [7] by optimizing the vectors $\vec{a}, \vec{a}^{\prime}, \vec{b}, \vec{b}^{\prime}$, the maximum possible average value of the Bell operator for the state $\rho$ is given by

$$
\max _{\mathcal{B}_{\mathrm{CHSH}}}\left|\operatorname{Tr}\left(\rho \mathcal{B}_{\mathrm{CHSH}}\right)\right|=2 \sqrt{\mathcal{M}(\rho)},
$$

where $\mathcal{M}(\rho)=\max _{j<k}\left\{h_{j}+h_{k}\right\} \leq 2$, and $h_{j}(j=$ $1,2,3)$ are the eigenvalues of the matrix $U=T^{T} T$ constructed from the correlation matrix $T$ and its transpose $T^{T}$. The $\mathrm{CHSH}$ inequality is violated if and only if $\mathcal{M}(\rho)>1$ [7]. In order to quantify the violation of the $\mathrm{CHSH}$ inequality, one can use $\mathcal{M}(\rho)$ or, equivalently,

$$
B(\rho) \equiv \sqrt{\max [0, \mathcal{M}(\rho)-1]}
$$

which yields $B=0$ if the $\mathrm{CHSH}$ inequality is not violated and $B=1$ for its maximal violation. In Sec. 6 we will describe how to measure the symmetric matrix $T^{T} T$ providing a method for efficient estimation of $B$ and $\mathcal{M}$.

\section{B. Entanglement measures}

In our considerations we apply three popular entanglement measures: negativity, concurrence and REE.

"Among all entanglement measures negativity arguably is the best known and most popular tool to quantify bipartite quantum correlations" [34]. The negativity for a bipartite state can be defined as [35, 36]:

$$
N(\rho)=\max \left[0,-2 \sum_{j} \mu_{j}\right]
$$

where the sum is taken over the negative eigenvalues $\mu_{j}$ of the partially transposed $\rho$ with respect to one of the subsystems, as denoted by $\rho^{\Gamma}$. In the case of two qubits, Eq. (7) simplifies to

$$
N(\rho)=\max \left[0,-2 \min \operatorname{eig}\left(\rho^{\Gamma}\right)\right]
$$

since $\rho^{\Gamma}$ has at most one negative eigenvalue in this case. The negativity is directly related to the logarithmic negativity, which has a direct physical meaning of the entanglement cost under PPT operations [10, 37. However, for convenience, we use the negativity instead.

For higher-dimensional systems, the negativity has another important interpretation as an estimator of entangled dimensions, i.e., how many degrees of freedom of two subsystems are entangled 34. We note that the dimension of Hilbert spaces can also be tested by the violations of Bell's inequality 38 .

The Wootters concurrence [11] is defined as

$$
C(\rho)=\max \left\{0,2 \max _{j} \lambda_{j}-\sum_{j} \lambda_{j}\right\}
$$

where $\left\{\lambda_{j}^{2}\right\}=\operatorname{eig}\left[\rho\left(\sigma_{2} \otimes \sigma_{2}\right) \rho^{*}\left(\sigma_{2} \otimes \sigma_{2}\right)\right]$. This measure is a monotonic and convex function of the entanglement of formation [12]. As in the case of the negativity and logarithmic negativity, it is often more convenient to operate with the concurrence instead of the entanglement of formation.

The REE is defined as

$$
E_{R}(\rho)=\min _{\sigma \in \mathcal{D}} S(\rho \| \sigma)=S\left(\rho \| \sigma_{0}\right),
$$

where $S(\rho \| \sigma)=\operatorname{Tr}\left(\rho \log _{2} \rho-\rho \log _{2} \sigma\right)$ is the relative entropy to be minimized over a set $\mathcal{D}$ of separable states $\sigma$ [8, 39]. The REE is used to distinguish a density matrix $\rho$ from the closest separable state (CSS) $\sigma_{0}$. For pure states, the REE reduces to the von Neumann entropy of one of the subsystems. However, the REE is not a true metric, because it is not symmetric and does not fulfill the triangle inequality. An analytical formula for $\sigma_{0}$ (and thus for the REE) for a given general two-qubit state $\rho$ is very unlikely to be found [40]. Nevertheless, there is a solution of the inverse problem [41. Probably, the most efficient numerical method for calculating the REE was described in Ref. 42 and, thus, it is used here.

\section{EXTREMAL ENTANGLEMENT FOR A GIVEN CHSH VIOLATION}

For each of the three entanglement measures listed in Sec. 2. we can ask about the states that are extremal, i.e., have the maximal or minimal value of one entanglement measure for a given fixed value of another entanglement measure [40, 43 46] or the CHSH violation measure [14, 15. Similarly, we state a more specific question about the maximal entanglement for vanishing of any other fixed degree of the CHSH violation. In this section we show 
that for all the above-mentioned entanglement measures, the states of the highest (lowest) entanglement for a given violation of the CHSH inequality are in fact the same class of states denoted as $\rho_{\max }\left(\rho_{\min }\right)$.

\section{A. Optimal amplitude damped states}

The amplitude-damped states can be defined by 15

$$
\rho(\alpha, p)=p\left|\psi_{\alpha}\right\rangle\left\langle\psi_{\alpha}|+(1-p)| 00\right\rangle\langle 00|,
$$

where $\left|\psi_{\alpha}\right\rangle=\sqrt{\alpha}|01\rangle+\sqrt{1-\alpha}|10\rangle$ with $p, \alpha \in[0,1]$. As discussed in detail in Ref. 15, these states can be obtained by subjecting pure states $\left|\psi_{\alpha^{\prime}}\right\rangle$ to amplitude damping. In the special case for $\alpha=1 / 2$, the state $\rho(\alpha, p)$ is referred to as the Horodecki state, which is a mixture of a Bell state (in our case, the singlet state) and a separable state orthogonal to it.

The amplitude-damped states, which provide the upper bound for the REE for a given value $B$ of the CHSH violation, are shown in Figs. 1 (bottom) and 2. Moreover, as found in Ref. 15, the amplitude-damped states $\rho(\alpha, p) \equiv \rho_{\max }\left(B_{0}\right)$, having the maximal value of the REE, $E_{R, \max }$, for a given value $B$, are the following

$$
\begin{aligned}
& p= \begin{cases}\frac{1}{4}\left(2+\sqrt{2+2 B^{2}}\right) & \text { if } B<B_{0}, \\
1 & \text { if } B>B_{0},\end{cases} \\
& 1 \geq p \geq \frac{1}{4}\left(2+\sqrt{2+2 B_{0}^{2}}\right) \text { if } B=B_{0},
\end{aligned}
$$

where $B_{0}=0.81686$. For the negativity and concurrence for a given value of $B$, the states $\rho_{\max }\left(B_{0}\right)$ are also optimal but for $B_{0}=1$ [so $p=\frac{1}{4}\left(2+\sqrt{2+2 B^{2}}\right)$ for any $B$ ] as shown in Sec. 4 and Appendix A respectively. Thus, the maximal values of the entanglement measures for a given value of $\xi^{2}=B^{2}+1$ are found as

$$
\begin{aligned}
& N_{\max }(\xi)=\frac{\sqrt{2}}{4}\left(\xi+\sqrt{5 \xi^{2}-2 \sqrt{2} \xi+2}\right)-\frac{1}{2} \\
& C_{\max }(\xi)=\frac{\sqrt{2} \xi^{2}+2 \xi}{2 \sqrt{\xi^{2}+2 \sqrt{2} \xi+2}}
\end{aligned}
$$

while $E_{R, \max }(B)$ is given in Ref. [15].

\section{B. Optimal phase damped states}

The lower bound on the three entanglement measures vs the CHSH violation $B$ is achieved by the Bell-diagonal states,

$$
\rho_{\mathrm{D}}=\sum_{i=1}^{4} \lambda_{i}\left|\beta_{i}\right\rangle\left\langle\beta_{i}\right|
$$
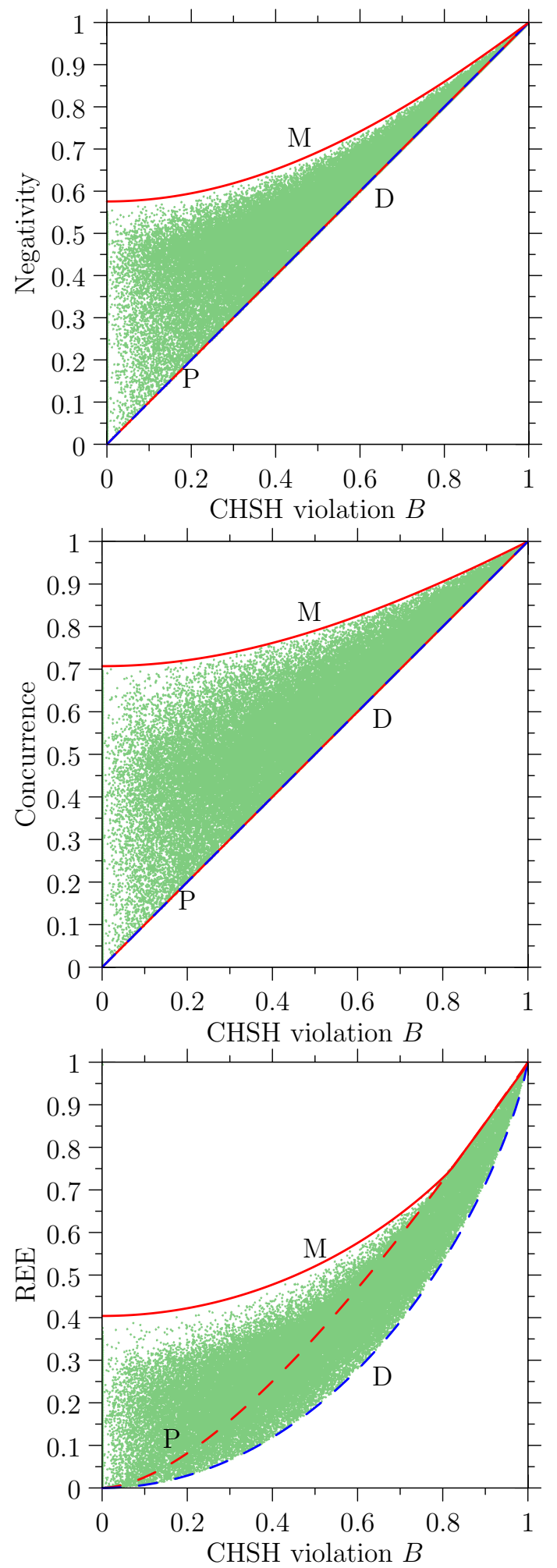

FIG. 1: (Color online) From the top: the negativity $N$, concurrence $C$, and relative entropy of entanglement $E_{R}$ versus the CHSH violation $B$ for $10^{6}$ random two-qubit states. The extremal states are marked as $\mathrm{P}$ for pure state, D for Belldiagonal states $\rho_{\min }$, and $\mathrm{M}$ for $\rho_{\max }$. The maximal values of $N_{\max }(B=0)=0.57567, C_{\max }(B=0)=0.70711$, and $E_{R, \max }(B=0)=0.404$ are reached for the M states $\rho_{\max }$. 


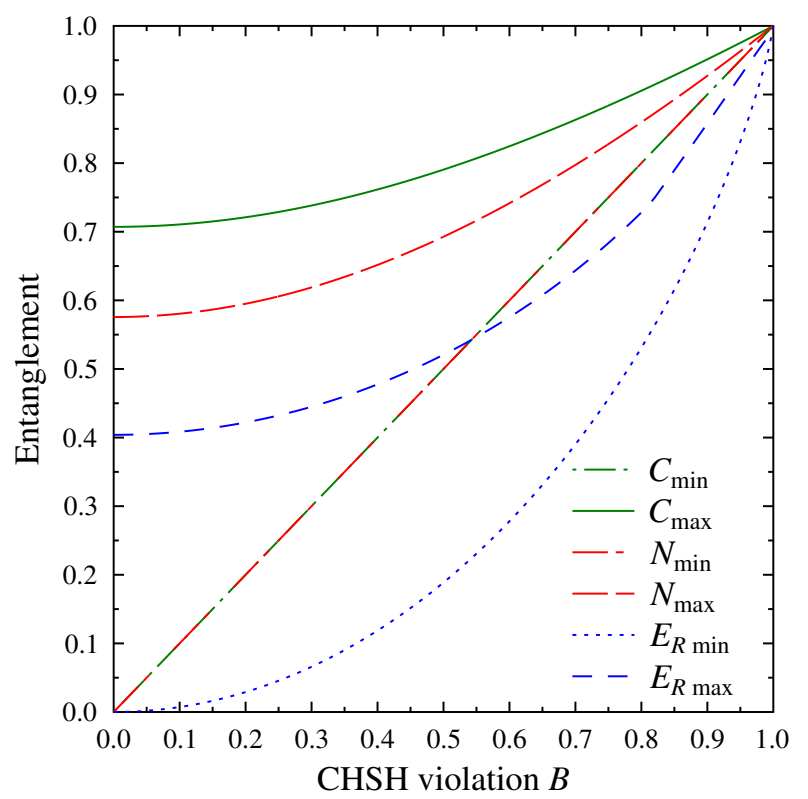

FIG. 2: (Color online) Comparison of the extremal values of the relative entropy of entanglement $E_{R}$, concurrence $C$, and negativity $N$ as functions of the $\mathrm{CHSH}$ violation $B$.

as labeled by D in Fig. 1. Here $\left|\beta_{i}\right\rangle$ are the Bell states and the parameters $\lambda_{j}$ are non-negative and normalized, $\sum_{i} \lambda_{i}=1$. The reason why the Bell-diagonal states provide the lower bound for $B$ given for a fixed function of the spectral properties of two-qubit density functions was explained in Ref. 14. The Bell-diagonal states can be produced by two-qubit pure states subjected to phase damping [15]. These states, assuming that $\lambda_{3}=\lambda_{4}=0$, can be given in terms of the CHSH violation degree $B$ as follows:

$$
\rho_{\min }=\frac{1}{2}\left[(1+B)\left|\beta_{1}\right\rangle\left\langle\beta_{1}|+(1-B)| \beta_{2}\right\rangle\left\langle\beta_{2}\right|\right],
$$

where $\left|\beta_{1}\right\rangle$ and $\left|\beta_{2}\right\rangle$ denote two orthogonal Bell states. Note that the relation between the CHSH violation and entanglement for the Bell-diagonal states is very simple, as given by

$$
\begin{aligned}
N_{\min }(B) & =C_{\min }(B)=B, \\
E_{R \min }(B) & =1-h[(1+B) / 2],
\end{aligned}
$$

where $h(x)=-x \log _{2} x-(1-x) \log _{2}(1-x)$ is the binary entropy.

\section{EXTREMALITY CONDITIONS FOR NEGATIVITY FOR A GIVEN CHSH VIOLATION}

Here we show that the amplitude-damped states given by Eq. (11), for the parameters:

$$
\begin{aligned}
p & =\frac{1}{4}(2+\sqrt{2} \xi), \\
\alpha & =\frac{1}{2}\left(1-\sqrt{1-\frac{4 \xi^{4}}{\left(\xi^{2}+\sqrt{2} \xi\right)^{2}}}\right),
\end{aligned}
$$

where $\xi^{2}=1+B^{2}$, are likely to provide the upper bound of the negativity $N$ for a given value $B$ of the CHSH violation. For this purpose we apply a generalized method of Lagrange multipliers and test the KKT conditions [13].

Thus, let us consider the following Lagrange function

$$
\begin{aligned}
\mathcal{L}= & B(\rho)+l\left[\frac{N}{2}-\operatorname{Tr}\left(\rho(|\psi\rangle\langle\psi|)^{\Gamma}\right)\right] \\
& -\operatorname{Tr}(X \rho)+\lambda(\operatorname{Tr} \rho-1),
\end{aligned}
$$

where $l, X$, and $\lambda$ are Lagrange multipliers, and $(|\psi\rangle\langle\psi|)^{\Gamma}$ is the optimal state for $\rho$ providing $N(\rho)=$ $-2 \operatorname{Tr}\left[(|\psi\rangle\langle\psi|)^{\Gamma} \rho\right]$.

The Lagrange function is stationary if it remains unchanged after an arbitrary small deviation of $\rho \rightarrow \rho+\Delta$, where $\Delta$ is defined on the support space of $\rho$. Thus, our Lagrange function

$$
\mathcal{L} \rightarrow \mathcal{L}+\operatorname{Tr}\left[\Delta\left(\mathcal{B}^{\prime}{ }_{\mathrm{CHSH}}+l(|\psi\rangle\langle\psi|)^{\Gamma}-X+\lambda\right)\right]
$$

should remain constant for small $\Delta$, i.e.,

$$
\begin{gathered}
\mathcal{B}^{\prime}{ }_{\mathrm{CHSH}}+l(|\psi\rangle\langle\psi|)^{\Gamma}-X+\lambda=0, \\
X \geq 0, \quad \operatorname{Tr}(X \rho)=0,
\end{gathered}
$$

where $\mathcal{B}^{\prime}{ }_{\mathrm{CHSH}}$ is the operator satisfying $B(\rho)=$ $\operatorname{Tr}\left(\rho \mathcal{B}^{\prime}{ }_{\mathrm{CHSH}}\right)$. Let us also note that $X \geq 0$ is required only for the eigenvalues in the support space of $\rho$.

Moreover, it follows from Eq. 22a), after taking the mean value for $\rho$, that

$$
\lambda=l \frac{N}{2}-B(\rho) .
$$

Thus, we can rewrite the KKT conditions as

$$
\begin{aligned}
& X=\mathcal{B}^{\prime}{ }_{\mathrm{CHSH}}-B(\rho)+l\left(\frac{N(\rho)}{2}+(|\psi\rangle\langle\psi|)^{\Gamma}\right) \geq 0, \\
& \operatorname{Tr}(X \rho)=0 .
\end{aligned}
$$

For the following rank-2 mixed states $\rho=\lambda_{1}\left|e_{1}\right\rangle\left\langle e_{1}\right|+$ $\lambda_{2}\left|e_{2}\right\rangle\left\langle e_{2}\right|$, which we conjecture to be extremal on the basis of our numerical simulation, we can easily derive the following expressions that can be used with the $l$ multiplier as

$$
\begin{aligned}
\left\langle e_{1}\left|\mathcal{B}^{\prime}{ }_{\mathrm{CHSH}}\right| e_{2}\right\rangle= & -l\left\langle e_{1}\left|(|\psi\rangle\langle\psi|)^{\Gamma}\right| e_{2}\right\rangle, \\
\left\langle e_{1}\left|\mathcal{B}^{\prime}{ }_{\mathrm{CHSH}}\right| e_{1}\right\rangle= & -l\left(\frac{N(\rho)}{2}+\left\langle e_{1}\left|(|\psi\rangle\langle\psi|)^{\Gamma}\right| e_{1}\right\rangle\right) \\
& +B(\rho) .
\end{aligned}
$$


By applying the KKT conditions we can check if a given state is optimal having its $\mathcal{B}^{\prime}{ }_{\mathrm{CHSH}}$ and $(|\psi\rangle\langle\psi|)^{\Gamma}$. The $\mathrm{CHSH}$ operator for the amplitude-damped states reads as

$$
\mathcal{B}^{\prime}{ }_{\mathrm{CHSH}}=\left\{\begin{array}{c}
\eta_{1}\left[(1-2 p) \sigma_{3}^{\otimes 2}+2 p \sqrt{(1-\alpha) \alpha} \sigma_{1}^{\otimes 2}-1\right] \\
\text { if } 4 p^{2}(1-\alpha) \alpha-(1-2 p)^{2}<0, \\
\eta_{2}\left[\begin{array}{c}
\left.2 p \sqrt{(1-\alpha) \alpha}\left(\sigma_{1}^{\otimes 2}+\sigma_{2}^{\otimes 2}\right)-1\right] \\
\text { otherwise, }
\end{array}\right.
\end{array}\right.
$$

where $\eta_{1}=1 / \sqrt{(1-2 p)^{2}+4 p^{2} \alpha(1-\alpha)-1}$ and $\eta_{2}=$ $1 / \sqrt{8 p^{2}(1-\alpha) \alpha-1}$, whereas

$$
|\psi\rangle=\mathcal{N}\left[\left(\sqrt{q^{2}+4 y^{2}}-1\right)|00\rangle+2 y|11\rangle\right],
$$

where $y=p \sqrt{\alpha(1-\alpha)}, q \equiv 1-p$, and $\mathcal{N}$ is a normalization constant.

The above results allow us to conclude that the optimal amplitude-damped states $\rho(\alpha, p)$, which maximize the negativity $N(\rho)$ for a given $B(\rho)$, are for the parameters $p$ and $\alpha$, given by Eq. (18). These parameters are the same as those resulting in the maximum REE for a fixed $B$ as given by Eq. (12) but with $B_{0}=1$.

Then the negativity for $\rho_{\max }(1)$ can be readily found as given by Eq. (13), which reaches its maximum $N_{\max } \approx$ 0.57567 for $B=0$. This result is confirmed by our Monte Carlo simulation shown in Fig. 1 (top).

Similar reasoning confirms that the minimal negativity is reached by the $\rho_{\min }$ states, given by Eq. (16).

\section{CONCURRENCE VS NEGATIVITY IF CHSH INEQUALITY IS SATISFIED}

One can conjecture that there is a direct relation between the concurrence and negativity for a fixed $\mathrm{CHSH}$ violation for the simple case of general two-qubit mixed states, which would then make the results of the former sections somewhat trivial. Here we show that there is no such relation for general two-qubit mixed states for a fixed $B=0$.

It is worth noting that even if a given entangled state does not violate any Bell type inequality, but still can be used for quantum teleportation, as shown by Popescu 47 on the example of Werner's entangled state given by Eq. 11) for $p \in(1 / 3,1 / \sqrt{2}$. Popescu concluded that: "The nonlocality responsible for violations of Bell's inequalities is not equivalent to that used in teleportation, although they probably are two aspects of the same physical property" 47]. However, it has recently turned out that these two aspects are in fact quite closely connected. Indeed, all entangled states useful for teleportation lead to deterministic violation of Bells inequality (so, they are nonlocal resources) as shown by Cavalcanti et al. [48] with the help of the phenomenon of superactivation of quantum nonlocality. Moreover, as demonstrated in Ref. [49, all bipartite entangled states are useful as

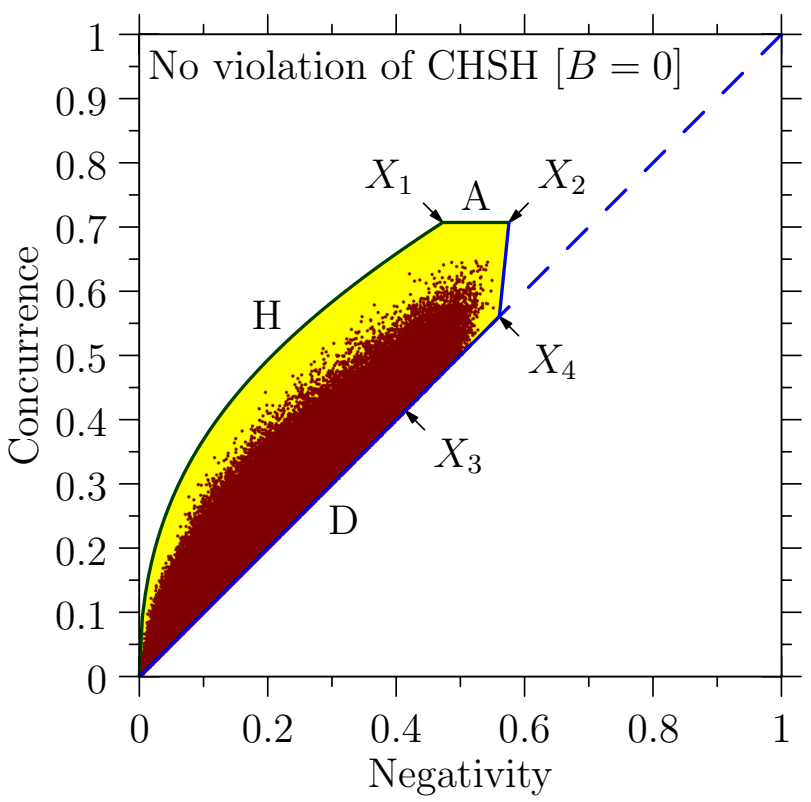

FIG. 3: (Color online) Concurrence $C$ vs negativity $N$ for two-qubit states satisfying the $\mathrm{CHSH}$ inequality $(B=0)$. All these states cover the region marked in bright yellow. States generated by our Monte Carlo simulations correspond to dark brown dots. The extremal states are marked as D for the Belldiagonal states, $\mathrm{H}$ for the Horodecki states, and $\mathrm{A}$ for the amplitude-damped states given by Eq. (11) with the proper choice of parameters $p, \alpha$. The points $\bar{X}_{k}=\left(N_{k}, C_{k}\right)$ and other details are specified in the text.

a nonclassical resource for quantum information processing.

Figure 3 shows the area covered by two-qubit states $\rho$ satisfying the CHSH inequality [i.e, $B(\rho)=0$ ] for the concurrence $C(\rho)$ plotted vs the negativity $N(\rho)$. The marked points $X_{k}=\left(N_{k}, C_{k}\right)$, with $k=1, \ldots, 4$, correspond to the following negativities:

$$
\begin{aligned}
& N_{1}=\frac{1}{\sqrt{2}}+\sqrt{2-\sqrt{2}}-1 \approx 0.4725 \\
& N_{2}=\frac{1}{4}(\sqrt{2}+\sqrt{14-4 \sqrt{2}}-2) \approx 0.5757,
\end{aligned}
$$

$N_{3}=\sqrt{2}-1 \approx 0.4142$, and $N_{4}=\frac{1}{4}(3 \sqrt{2}-2) \approx 0.5607$, as well as to the concurrences: $C_{1}=C_{2}=1 / \sqrt{2} \approx 0.7071$, and $C_{k}=N_{k}$ for $k=3,4$. The upper bound of this area for $N \in\left[0, N_{1}\right]$ is given by

$$
C(N)=\sqrt{2 N(N+1)}-N,
$$

or, equivalently, by $N(C)=\sqrt{(1-C)^{2}+C^{2}}-(1-C)$. This bound can be reached by the Horodecki states (labeled by $\mathrm{H}$ ), given by Eq. (11) for $\alpha=1 / 2$ and $p=C(\rho)$. We note that the upper bound of the concurrence vs negativity without specifying the CHSH violation is also given by Eq. (29) but for the whole range $0 \leq N \leq 1$ [43, 45]. The upper bound for $N \in\left[N_{1}, N_{2}\right]$ is 
likely to be $C(N)=1 / \sqrt{2}$, which can be reached by the amplitude-damped states (labeled by A) given by Eq. 111 for $p=1 /[2 \sqrt{2 \alpha(1-\alpha)}]$ and $\alpha=\left[\alpha_{-}, \alpha_{+}\right]$, with $\alpha_{ \pm}=1 / 2 \pm \sqrt{8 \sqrt{2}-11}$. The lower bound for $N \in\left[0, N_{4}\right]$ is simply given by $C=N$ and can be reached by the rank- 4 Bell-diagonal states, given by Eq. 15) for $\lambda_{1}=\lambda_{2}=\lambda_{3}=(1-N) / 6$ and, thus, $\lambda_{4}=\max _{n} \lambda_{n}=(1+N) / 2$. The lower bound for $N \in\left[0, N_{3}\right]$ can also be reached by the rank-3 Belldiagonal states with $\lambda_{1}=0, \lambda_{2}=\lambda_{3}=(1-N) / 4$, and $\lambda_{4}$ being the same as in the previous case. The rank-2 Bell-diagonal states satisfying $B=0$ correspond only to the point $C=N=0$. It is also worth noting that the lower bound of $C$ vs $N$ for arbitrary $B$ is also simply given by $C=N$ but for any $0 \leq N \leq 1$ [43, 45]. We could not find analytical examples of states corresponding to the lower bound for $N \in\left[N_{4}, N_{2}\right]$. Note that it is a very narrow region, as equal to $N_{2}-N_{4}=0.015$, so it is even difficult to numerically simulate states satisfying both $N \in\left(N_{4}, N_{2}\right)$ and $B=0$. Of course, one can analyze, e.g., the mixture $\rho_{q}=q \rho_{X_{2}}+(1-q) \rho_{X_{4}}$, where $0 \leq q \leq 1$, of the discussed Bell-diagonal state at point $X_{4}$ and the amplitude-damped state at point $X_{2}$. Then one can observe that only for $q \geq 0.9893 \ldots$ is the negativity $N\left(\rho_{q}\right) \geq N_{4}$, which corresponds to the concurrence $C\left(\rho_{q}\right) \geq 0.6706>C_{4}$. This explicitly shows that the state $\rho_{q}$ can have the negativity $N \in\left(N_{4}, N_{2}\right)$ but it is not the lower bound of $C$ vs $N$ for $q>0$.

A closer analysis of Fig. 3 also shows that there are infinitely many pairs of two-qubit states (say, $\rho_{1}$ and $\rho_{2}$ ) violating the following intuitive conditions for ordering states with the concurrence and negativity:

$$
\begin{aligned}
& N\left(\rho_{1}\right)=N\left(\rho_{2}\right) \Leftrightarrow C\left(\rho_{1}\right)=C\left(\rho_{2}\right) \\
& N\left(\rho_{1}\right)>N\left(\rho_{2}\right) \Leftrightarrow C\left(\rho_{1}\right)>C\left(\rho_{2}\right)
\end{aligned}
$$

for a fixed CHSH violation $B\left(\rho_{1}\right)=B\left(\rho_{2}\right)$. Of course, there are also infinitely many other states satisfying these conditions. In particular, by analyzing Fig. 3, one can find analytical nontrivial examples of states which satisfy the CHSH inequality and are ordered differently by these entanglement measures, e.g.,

$$
\begin{aligned}
& N\left(\rho_{X_{1}}\right)<N\left(\rho_{X_{4}}\right) \quad \text { and } \quad C\left(\rho_{X_{1}}\right)>C\left(\rho_{X_{4}}\right), \\
& N\left(\rho_{X_{1}}\right)=N\left(\rho_{X_{5}}\right) \quad \text { and } \quad C\left(\rho_{X_{1}}\right)>C\left(\rho_{X_{5}}\right), \\
& N\left(\rho_{X_{1}}\right)<N\left(\rho_{X_{2}}\right) \quad \text { and } \quad C\left(\rho_{X_{1}}\right)=C\left(\rho_{X_{2}}\right),
\end{aligned}
$$

where $\rho_{X_{5}}$ is, e.g., the Bell-diagonal state with $\lambda_{1}=\lambda_{2}=$ $\lambda_{3}=\left(1-N_{1}\right) / 6, \lambda_{4}=\max _{n} \lambda_{n}=\left(1+N_{1}\right) / 2$, and $N_{1}$ the same as for $\rho_{X_{1}}$. Of course, one can also identify pairs of states, e.g., $\rho_{X_{2}}$ and $\rho_{X_{4}}$, which are ordered in the same way, e.g., $N\left(\rho_{X_{2}}\right)>N\left(\rho_{X_{4}}\right)$ and $C\left(\rho_{X_{2}}\right)>C\left(\rho_{X_{4}}\right)$.

This relativity of ordering states by different entanglement measures is a well-known phenomenon [16, 19, 4446, 50,52, which clearly shows the lack of simple relations of, e.g., the negativity and concurrence (when the degree of the $\mathrm{CHSH}$ violation is irrelevant) for two-qubit

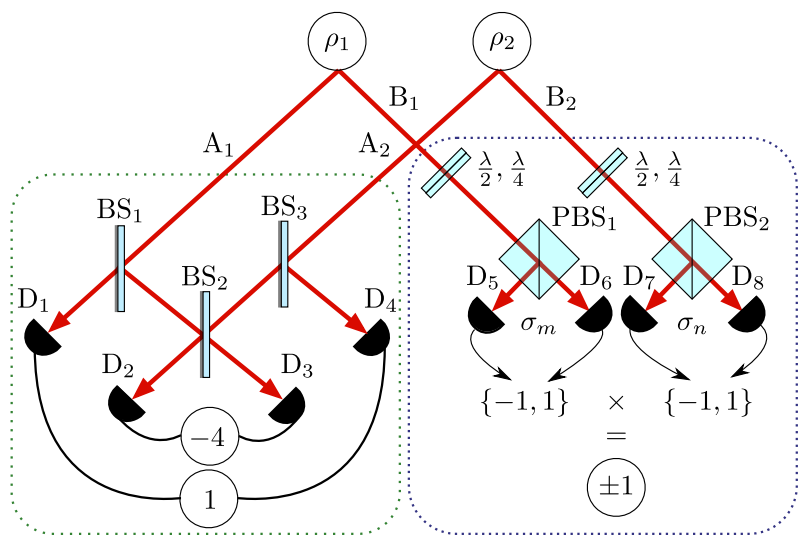

FIG. 4: (Color online) Setup implementing the measurement of $\left(T^{T} T\right)_{m, n}$ using two sources (or a single photon source with routing and delaying every second pair of photons) of a two-qubit state $\left(\rho_{1}\right.$ and $\left.\rho_{2}\right)$. The basic building blocks are as follows: beam splitters (BSs), polarizing beam splitters (PBSs), quarter-wave plate $(\lambda / 4)$ and half-wave plate $(\lambda / 2)$, and standard detectors. The values of $m, n=x, y, z$ are set by rotating the polarization by means of the wave plates, i.e., one $\lambda / 4$ and one $\lambda / 2$ plate, where $\lambda$ is the wavelength. Circled -4 (and \pm 1 ) means that this value is assigned if the corresponding detectors (D) click. Since the investigated function of the correlation matrix $T$ is symmetric we need only to measure it in six configurations, e.g, $(m, n)=(x, x),(x, y),(x, z),(y, y),(y, z),(z, z)$. Due to the probabilistic nature of the path taken by photons after the BS interaction, the setup gives a conclusive result in half of the cases if $\rho_{1}$ and $\rho_{2}$ are supplied at the input.

states. Here we showed the relativity of ordering states by the negativity and concurrence for a fixed value of the CHSH violation.

\section{PROPOSAL OF EFFICIENT MEASUREMENT OF THE CORRELATION MATRIX $T^{T} T$}

Knowing the lower and upper bounds of the three entanglement measures for a given $\mathrm{CHSH}$ violation $B$, we are now able to deduce the range of entanglement of any two-qubit state for a fixed $B$. The expression for the CHSH violation $B$ depends solely on the eigenvalues of the symmetric real matrix $T^{T} T$. Here we present an efficient method for measuring this correlation matrix $T^{T} T$.

We can express the elements of this matrix using two copies $\rho_{1}$ and $\rho_{2}$ of the two-qubit state $\rho$ as

$$
\left(T^{T} T\right)_{m, n}=\operatorname{Tr}\left[\left(\rho_{A_{1} B_{1}} \otimes \rho_{A_{2} B_{2}}\right) U_{A_{1} A_{2}} \otimes\left(\sigma_{m} \otimes \sigma_{n}\right)_{B_{1} B_{2}}\right],
$$

where $\rho_{A_{1} B_{1}} \equiv \rho_{1}$ and $\rho_{A_{2} B_{2}} \equiv \rho_{2}$ for the subsystems $A$ and $B$, whereas the operator $U_{A_{1} A_{2}}=$ $\left(-4\left|\Psi^{-}\right\rangle\left\langle\Psi^{-}\right|+I\right)_{A_{1} A_{2}}$ is given in terms of the singlet projection $\left|\Psi^{-}\right\rangle\left\langle\Psi^{-}\right|$onto the corresponding subsystems and the two-qubit identity operation $I$ (for a derivation see Appendix B). Since the $3 \times 3$ matrix $T^{T} T$ is symmet- 
ric, $\left(T^{T} T\right)_{m, n}=\left(T^{T} T\right)_{n, m}$, so it is completely defined by six real numbers, which can be directly measured for, e.g., single-photon polarization qubits. We choose, e.g., $|0\rangle(|1\rangle)$ to represent a horizontally (vertically) polarized photon. For such qubits, $T^{T} T$ can be measured by the setup shown in Fig. 4. The left-hand-side module of this setup, which consists of three 50:50 asymmetric beam splitters (BSs), performs the measurement of the $U_{A 1 A 2}$ operator. The operation of this module was described in detail (considering imperfections including finite detection efficiency) in Ref. [53. The possible outcomes for a single measurement instance $a_{k}$ are $a_{k} \in\{-4,-1,0,1,4\}$, which are the products of the outcomes $-4,0,1$ corresponding to a particular coincidence detection in the module 1 , for a coincidence detection in the detectors $\mathrm{D}_{1}$ and $\mathrm{D}_{4} ;-4$, for a coincidence detection in the detectors $\mathrm{D}_{2}$ and $\mathrm{D}_{3}$; and 0 , if neither of the two coincidences has been detected. Moreover, the right-hand-side module of the setup in Fig. 4 measures the product $\sigma_{m} \otimes \sigma_{n}$. The outcomes $-1,1$ of this module occur for measuring the product of the Pauli matrices. The useful values of $a_{n} \neq 0$ appear for one-half of the cases when the states $\rho_{1}$ and $\rho_{2}$ are delivered and assuming perfect detectors. For realistic components (see the analysis in Ref. [53]), this setup would provide us with a good estimation of $T^{T} T$ in a time period corresponding to switching between the six settings of $\sigma_{m} \otimes \sigma_{n}$ instead of nine settings required for the full tomography of the $T$ matrix. The expected values obtained read

$$
\left\langle\sigma_{m} \otimes \sigma_{n}\right\rangle=\frac{1}{K_{0}} \sum_{k=1}^{K} a_{k},
$$

where $K$ is the number of measurements, $K_{0}=$ $\sum_{k=1}^{K} \delta_{\left|a_{k}\right|, 1}$ and $\delta_{\left|a_{k}\right|, 1}$ is the Kronecker $\delta$. Note that the depicted measurement method is not limited to measuring $B$ for any two-qubit state. It measures $T^{T} T$, which contains more information than the sum of the two largest eigenvalues used for calculating $B$.

\section{A. Proposal for experimental optimization}

Here we discuss an optimization of the setup to make it experimentally more feasible. Our implementation of the left-hand-side module, depicted conceptually in Fig. 4. requires three balanced beam splitters and four detectors. From the experimentalist point of view, the larger the number of components, the larger the measurement error. For example, the splitting ratio of beam splitters is particularly sensitive to mount alignment and manufacturing precision. Furthermore all the detectors have to be calibrated to the same relative detection efficiency.

To reduce the number of required optical components, we propose a modified measurement setup depicted in Fig. 5. As the conceptual setup in Fig. 4 shows, there are two distinct measurement regimes in the module. The first regime is implemented by two-photon overlap on a

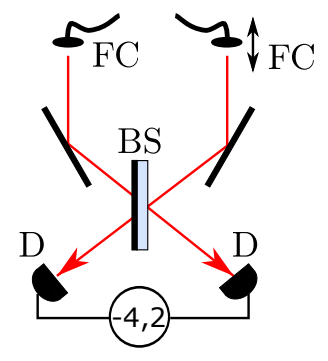

FIG. 5: (Color online) Experiment-friendly setup replacing the left-hand side measurement module in Fig. 4 BS, balanced beam splitter; FC, fiber coupler; D, detector. Motorized translation (marked by double arrow) is used to tune the temporal delay between the photons in order to switch between measurement regimes as explained in the text.

balanced beam splitter projecting the state onto the singlet state. The corresponding coincidence rate is then multiplied by the factor of -4 . The second regime is just a plain coincidence count (detectors $\mathrm{D}_{1}$ and $\mathrm{D}_{4}$ ). In the modified setup, we implement both these regimes using a single beam splitter. To switch between the regimes, we suggest a delay line to tune the temporal overlap between the interacting photons. The first measurement regime is obtained by setting the delay between the photons to zero, while the second regime is obtained when the delay is sufficiently larger than a single-photon coherence length. In the second regime, the two photons impinge on the beam splitter independently and they exit by different output ports in half of the cases only. For this reason, this number of coincidences has to be multiplied by 2 to implement the conceptual setup.

The benefits of the optimized setup are at least threefold: (i) The number of beam splitters is reduced by a factor of 3. (ii) Only one pair of detectors is used, so there is no need for the calibration within the module. The only calibration to be performed is the mutual calibration of the efficiencies of the detector pairs across the left- and right-hand-side modules of the setup. (iii) Another minor benefit of the modified version of the setup is that it can be constructed using a standardized twophoton-state characterization device [54] routinely used in other experiments. Note that since there is need for singlet-state projection even in the original setup, such a delay line would be needed in order to stabilize the setup anyway. Therefore it does not impose any additional experimental requirements.

\section{CONCLUSIONS}

We have analyzed, as summarized in Fig. 2, the relation between the Horodecki measure of the CHSH inequality violation (or single-copy nonlocality) and three common entanglement measures: the negativity, concurrence, and relative entropy of entanglement. We discovered optimal states that provide the upper bound on the 
entanglement measures for a given CHSH violation. We provided both numerical and analytic evidence by testing the KKT extremality conditions within a generalized Lagrange multiplier method in the case of the negativity for a given CHSH violation. We also checked that the states found satisfy the Verstraete-Wolf conditions [14] for the extremal concurrence for a given $\mathrm{CHSH}$ violation. Remarkably, the states belong to the same class of states for all the investigated measures of entanglement, including the REE. We showed that the states providing the upper and lower bounds on the entanglement measures for a given value of the CHSH violation can be simply obtained by the amplitude and phase damping of pure states, respectively. We also found extremal states for the concurrence versus negativity for a fixed value of the $\mathrm{CHSH}$ violation (i.e., $B=0$ ).

Moreover, we described a method to efficiently measure the correlation matrix $T^{T} T$, and, thus, to estimate the Horodecki degree of the CHSH violation. This method together with the found bounds on the entanglement measures discussed provides an easy and practical way of estimating entanglement for arbitrary two-qubit states with a fixed degree of $\mathrm{CHSH}$ violation.

It is worth comparing our method with the standard methods, in which the violation of the CHSH inequality can be tested using four correlation measurements. Hence, one could ask about the advantage in estimating (in a non device-independent way) a correlation matrix using six correlation measurements. One might think that we use more measurements to achieve less. However, this four-measurement approach refers just to testing the $\mathrm{CHSH}$ violation for a given state and for given positions of analyzers. In contrast, our work is about quantifying the CHSH violation for a given state by optimizing over all possible positions of analyzers to have the greatest degree of the CHSH violation. This approach requires more measurements than in the case of ordinary unoptimized measurements of the CHSH violation. Namely, our approach is based on the Horodecki measure of the $\mathrm{CHSH}$ violation corresponding to finding eigenvalues of a real symmetric $3 \times 3$ matrix with six independent unknown parameters for a given two-qubit state. Thus, one can conjecture that the minimum number of optical measurements is six, at least, if two copies of the state are simultaneously available [55]. If only one copy is available at a given moment, then the required number of measurements is even higher (arguably, equal to nine [55]).

Both upper and lower bounds are operationally important especially in relation to secure quantum communication (for a related study of secure quantum teleportation see, e.g., Ref. [56]). For example, let us assume that the degree of CHSH violation (including the case of no violation) of a given state $\rho$ is known. Then by applying our negativity bounds, we can calculate the bounds on the PPT-entanglement cost, which is the asymptotic number of maximally entangled states that are required to create the state $\rho$ under operations preserving the posi- tivity of the partial transpose. Analogously, by applying the Verstraete-Wolf concurrence bounds, one can calculate the bounds on the entanglement of formation.

It is worth noting that by measuring the correlation matrix $T$ of an arbitrary unknown two-qubit state, we can find directly the optimal measurement settings by applying the Horodecki theorem [7]. In contrast, by measuring $T^{T} T$ of a general state, we can determine the value of the CHSH violation optimized over all possible measurement settings without knowing these optimal settings explicitly. Only for a limited class of states, including those with symmetric $T$, the optimal settings can be determined completely from the $T^{T} T$ matrix.

Finally, we mention one possible application of our results. Recently, the CHSH inequality has been proved extremely useful for verifying the quantumness of a black box device (say, a claimed quantum computer) programmed to win the so-called CHSH game [57. Thus, with the help of our results, by looking at the results of the CHSH game, we are able to estimate how much entanglement was used by the tested black box.

\section{Acknowledgments}

We thank Paweł Horodecki, Yeong-Cherng Liang, and Satoshi Ishizaka for discussions. This work was supported by the Polish National Science Center under Grants No. DEC-2011/03/B/ST2/01903 and No. DEC-2011/02/A/ST2/00305. K. B. gratefully acknowledges support by the Operational Program Research and Development for Innovations European Regional Development Fund (Project No. CZ.1.05/2.1.00/03.0058) and the Operational Program Education for Competitiveness - European Social Fund (Project No. CZ.1.07/2.3.00/20.0017 and No. CZ.1.07/2.3.00/30.0041) of the Ministry of Education, Youth and Sports of the Czech Republic. K. L. acknowledges the support by the Czech Science Foundation (Project No. 13-31000P).

\section{Appendix A: States with extremal concurrence for a given CHSH violation}

The conditions satisfied by the extremal amount of the CHSH violation for a fixed value of the concurrence were given by Verstraete and Wolf in Ref. [14]. Note that, for quantifying the CHSH violation, the authors of Ref. 14 used the parameter $\beta=2 \sqrt{B^{2}+1}$. So, the CHSH inequality is satisfied for $\beta \leq 2$. Nevertheless, their results are valid also in our case since $B$ is uniquely determined by $\beta$. In order to solve the optimization problem, the method of Lorentz transformations on the extended correlation matrix $T$ was used in Ref. [14 to generate states of constant concurrence. It was found that pure and Bell-diagonal states have the maximal concurrence for a given value $B$ of $\mathrm{CHSH}$ violation, while the lowest con- 
currence for a given $B$ is achieved by, e.g., a mixture of a Bell state and a separable state orthogonal to it (the so-called Horodecki state). A summary of these results is shown in Fig. 1(middle).

The optimality of the states $\rho_{\max }$, given by Eqs. (11) and (18), for the whole range of $B$ can be demonstrated using the optimality conditions given in Ref. 14. This is straightforward since the matrix $R_{m, n}=\left\langle\sigma_{m} \otimes \sigma_{n}\right\rangle$ (for $m, n=0,1,2,3$, where $\sigma_{0}$ is the identity), which was used for testing the optimality conditions in Ref. [14], has the same structure as $\rho_{\max }$. The relevant parameters as defined in Ref. 14 read

$$
\begin{aligned}
a^{( \pm)} & =-\frac{\sqrt{2}\left(\xi^{2}-2\right)}{4(\xi+\sqrt{2})} \pm \frac{\sqrt{2}}{4} \sqrt{\xi^{2}+2 \sqrt{2} \xi+2,},(\mathrm{~A} 1 \mathrm{a}) \\
x & =y=\frac{\sqrt{2}}{2} \xi, \quad z=-\frac{\sqrt{2} \xi+\xi^{2}}{\sqrt{2} \xi+2}, \quad(\mathrm{~A} 1 \mathrm{~b})
\end{aligned}
$$

where $\xi^{2}=B^{2}+1$. These parameters satisfy the optimality conditions (in fact, they saturate the last two):

$$
\begin{aligned}
-1 & \leq z \leq 1 \\
(1+z)^{2}-\left(a^{(+)}+a^{(-)}\right)^{2} & \geq(x-y)^{2} \\
(1-z)^{2}-\left(a^{(+)}-a^{(-)}\right)^{2} & \geq(x+y)^{2} .
\end{aligned}
$$

Moreover, the concurrence for amplitude-damped states is

$$
C(\alpha, p)=2 p \sqrt{\alpha(1-\alpha)}
$$

so, in the case of extremal states, it can be expressed by Eq. (14). Thus, the states $\rho_{\max }$ belong to the class of states having the highest concurrence for a given degree $B$ of the $\mathrm{CHSH}$ violation reaching the maximum
$C_{\max }(\xi=1) \equiv C_{\max }(B=0)=1 / \sqrt{2}$ as shown in Fig. 11(middle).

\section{Appendix B: Two-copy formula for correlation matrix $T^{T} T$}

Here we derive a two-copy formula for the correlation matrix $T^{T} T$, given by Eq. 31, which is useful for our experimental proposal.

In the following, we use the Einstein summation convention. Let us start by recalling that we can express $T_{m n}$ as an expectation value of the Pauli matrices, i.e.,

$$
T_{m n}=\operatorname{Tr}\left[\left(\sigma_{m} \otimes \sigma_{n}\right) \rho\right]
$$

hence

$$
\begin{aligned}
\left(T^{T} T\right)_{m n} & =T_{k m} T_{k n}=\operatorname{Tr}\left[\left(\sigma_{k} \otimes \sigma_{m} \otimes \sigma_{k} \otimes \sigma_{n}\right)(\rho \otimes \rho)\right] \\
& =\operatorname{Tr}\left[\left(\sigma_{k} \otimes \sigma_{k}\right) \otimes\left(\sigma_{m} \otimes \sigma_{n}\right)(\rho \otimes \rho)^{\prime}\right] \\
& =\operatorname{Tr}\left\{\left[U_{A_{1} A_{2}} \otimes\left(\sigma_{m} \otimes \sigma_{n}\right)_{B_{1} B_{2}}\right]^{\prime}(\rho \otimes \rho)\right\},(\mathrm{B} 2)
\end{aligned}
$$

where $(\rho \otimes \rho)^{\prime}=S_{A_{2} B_{1}}(\rho \otimes \rho) S_{A_{2} B_{1}}, U=\sigma_{k} \sigma_{k}=$ $I-4\left|\Psi^{-}\right\rangle\left\langle\Psi^{-}\right|$, and $\left|\Psi^{-}\right\rangle$denotes the singlet state. The unitary transformation $S_{A_{2} B_{1}}=I \otimes S \otimes I$ swaps the modes $A_{2}$ and $B_{1}$, which can be given in terms of the swap operator

$$
S=\left(\begin{array}{llll}
1 & 0 & 0 & 0 \\
0 & 0 & 1 & 0 \\
0 & 1 & 0 & 0 \\
0 & 0 & 0 & 1
\end{array}\right)
$$

Equation B2 finally results in Eq. 31.
[1] A. Einstein, B. Podolsky, and N. Rosen, Phys. Rev. 47, 777 (1935).

[2] R. Horodecki, P. Horodecki, M. Horodecki, and K. Horodecki, Rev. Mod. Phys. 81, 865 (2009).

[3] J. S. Bell, Speakable and Unspeakable in Quantum Mechanics (Cambridge Univeristy Press, Cambridge, U. K., 2004).

[4] J. F. Clauser, M. A. Horne, A. Shimony, and R. A. Holt, Phys. Lett. A 23, 880 (1969).

[5] N. Brunner, D. Cavalcanti, S. Pironio, V. Scarani, and S. Wehner, arXiv:1303.2849.

[6] R. F. Werner, Phys. Rev. A 40, 4277 (1989).

[7] R. Horodecki, P. Horodecki, and M. Horodecki, Phys. Lett. A 200, 340 (1995); R. Horodecki, ibid. 210, 223 (1996).

[8] V. Vedral, M. B. Plenio, M. A. Rippin, and P. L. Knight, Phys. Rev. Lett. 78, 2275 (1997).

[9] A. Peres, Phys. Rev. Lett. 77, 1413 (1996); M. Horodecki, P. Horodecki, and R. Horodecki, Phys. Lett. A 223, 1 (1996).
[10] K. Audenaert, M. B. Plenio, and J. Eisert, Phys. Rev. Lett. 90, 027901 (2003).

[11] W. K. Wootters, Phys. Rev. Lett. 80, 2245 (1998).

[12] C. H. Bennett, D. P. DiVincenzo, J. A. Smolin, and W. K. Wootters, Phys. Rev. A 54, 3824 (1996).

[13] S. Boyd and L. Vandenberghe, Convex Optimization, (Cambridge University Press, Cambridge, U. K., 2004).

[14] F. Verstraete and M. M. Wolf, Phys. Rev. Lett. 89 170401 (2002).

[15] B. Horst, K. Bartkiewicz, and A. Miranowicz, Phys. Rev. A 87, 042108 (2013).

[16] S. Virmani and M. B. Plenio, Phys. Lett. A 268, 31 (2000).

[17] L. Derkacz and L. Jakóbczyk, Phys. Rev. A 72, 042321 (2005).

[18] L. Jakóbczyk and A. Jamróz, Phys. Lett. A 318, 318 (2003).

[19] A. Miranowicz, Phys. Lett. A 327, 272 (2004).

[20] L. Derkacz and L. Jakóbczyk, Phys. Lett. A 328, 26 (2004). 
[21] X. P. Liao, M. F. Fang, X. J. Zheng, and J. W. Cai, Phys. Lett. A 367, 436 (2007).

[22] A. G. Kofman and A. N. Korotkov, Phys. Rev. A 77, 052329 (2008).

[23] D. L. Deng and J. L. Chen, Int. J. Quantum Inf. 7, 1313 (2009).

[24] L. Mazzola, B. Bellomo, R. Lo Franco, and G. Compagno, Phys. Rev. A 81, 052116 (2010); B. Bellomo, G. Compagno, A. D'Arrigo, G. Falci, R. Lo Franco, and E. Paladino, Int. J. Quantum Inf. 9, 63 (2011).

[25] K. Berrada, Y. Hassouni, and H. Eleuch, Commun. Theor. Phys. 56, 679 (2011).

[26] M. L. Hu, Quantum Inf. Process. 12, 229 (2013).

[27] T. Moroder, J. D. Bancal, Y. C. Liang, M. Hofmann, and O. Gühne, Phys. Rev. Lett. 111, 030501 (2013).

[28] Y. C. Liang, T. Vertesi, and N. Brunner, Phys. Rev. A 83, 022108 (2011).

[29] E. Shchukin and W. Vogel, Phys. Rev. Lett. 95, 230502 (2005); A. Miranowicz and M. Piani, ibid. 97, 058901 (2006); H. Häseler, T. Moroder, and N. Lütkenhaus, Phys. Rev. A 77, 032303 (2008); A. Miranowicz, M. Piani, P. Horodecki, and R. Horodecki, ibid. 80, 052303 (2009).

[30] W. Vogel, Phys. Rev. Lett. 100, 013605 (2008); A. Miranowicz, M. Bartkowiak, X. Wang, Y. X. Liu, and F. Nori, Phys. Rev. A 82, 013824 (2010).

[31] T. Vertesi, Phys. Rev. A 78, 032112 (2008).

[32] C. Śliwa, Phys. Lett. A 317, 165 (2003); D. Collins and N. Gisin, J. Phys. A: Math. Gen. 37, 1775 (2004).

[33] B. Bellomo, R. Lo Franco, and G. Compagno, Phys. Lett. A 374, 3007 (2010).

[34] C. Eltschka and J. Siewert, Phys. Rev. Lett. 111, 100503 (2013).

[35] K. Życzkowski, P. Horodecki, A. Sanpera, and M. Lewenstein, Phys. Rev. A 58, 883 (1998).

[36] G. Vidal and R. F Werner, Phys. Rev. A 65, 032314 (2002).
[37] S. Ishizaka, Phys. Rev. A 69, 020301(R) (2004).

[38] N. Brunner, S. Pironio, A. Acin, N. Gisin, A. A. Methot, and V. Scarani, Phys. Rev. Lett. 100, 210503 (2008).

[39] V. Vedral and M. B. Plenio, Phys. Rev. A 57, 1619 (1998).

[40] A. Miranowicz, S. Ishizaka, B. Horst, and A. Grudka, Phys. Rev. A 78, 052308 (2008).

[41] S. Ishizaka, Phys. Rev. A 67, 060301(R) (2003); A. Miranowicz and S. Ishizaka, ibid. 78, 032310 (2008).

[42] Y. Zinchenko, S. Friedland, and G. Gour, Phys. Rev. A 82, 052336 (2010).

[43] F. Verstraete, K. M. R. Audenaert, J. Dehaene, and B. De Moor, J. Phys. A 34, 10327 (2001).

[44] T. C. Wei, K. Nemoto, P. M. Goldbart, P. G. Kwiat, W. J. Munro, and F. Verstraete, Phys. Rev. A 67, 022110 (2003).

[45] A. Miranowicz and A. Grudka, Phys. Rev. A 70, 032326 (2004),

[46] A. Miranowicz and A. Grudka, J. Opt. B: Quantum Semiclass. Opt. 6, 542 (2004).

[47] S. Popescu, Phys. Rev. Lett. 72, 797 (1994).

[48] D. Cavalcanti, A. Acin, N. Brunner, and T. Vértesi, Phys. Rev. A 87, 042104 (2013).

[49] L. Masanes, Phys. Rev. Lett. 96, 150501 (2006).

[50] J. Eisert and M. Plenio, J. Mod. Opt. 46, 145 (1999).

[51] K. Życzkowski, Phys. Rev. A 603496 (1999).

[52] A. Miranowicz, J. Phys. A 37, 7909 (2004).

[53] K. Bartkiewicz, K. Lemr, A. Černoch, and J. Soubusta, Phys. Rev. A 87, 062102 (2013).

[54] E. Halenková, A. Černoch, K. Lemr, J. Soubusta, and S. Drusová, Appl. Opt. 51, 474 (2012).

[55] P. Horodecki (private communication).

[56] S. K. Özdemir, K. Bartkiewicz, Y. X. Liu, and A. Miranowicz, Phys. Rev. A 76, 042325 (2007).

[57] B. W. Reichardt, F. Unger, and U. Vazirani, Nature (London) 496, 456 (2013). 